\title{
REVIEW
}

\section{Amyotrophic Lateral Sclerosis and Parkinsonism-dementia Complex on Guam: Immunohistochemical Studies}

\author{
Asao Hirano \\ Department of Pathology (Neuropathology), Montefiore Medical Center, Bronx, NY, USA
}

(Received for publication on October 14, 1991)

\begin{abstract}
The incidence of amyotrophic lateral sclerosis (ALS) and Parkinsonism-dementia complex (PDC) among the Chamorros in Guam is remarkably high. The patients with ALS have clinical and pathological characteristics similar to those in other parts of the world. The PDC patients display parkinsonism and progressive dementia and show a characteristic neuronal loss in certain parts of the central nervous system such as the hippocampus and substantia nigra. The Guamanian patients with ALS and PDC commonly have widespread Alzheimer's neurofibrillary changes, but without the associted senile plaques. We have applied immunohistochemical procedures to examine the expression of marker substances in Guamanian ALS and PDC. The markers studied include tau protein, ubiquitin, beta proteins, synaptophysin, calcineurin, Met-enkephalin, substance $P$ and tyrosine hydroxylase. The results were compared with the findings in patients with Alzheimer's disease, Parkinson's disease, sporadic ALS and familial ALS. (Keio J Med 41 (1): 6-9, March 1992)
\end{abstract}

Key words: immunohistochemistry, neurofibrillary changes, senile plaques

\section{Introduction}

The island of Guam in the Western Pacific represents a geographic isolate with a striking incidence of fatal neurological diseases. ${ }^{1}$ Among them, the best known ailments are amyotrophic lateral sclerosis (ALS) and parkinsonism-dementia complex (PDC) which affected the native adult Chamorro population. The clinicopathological features of ALS are essentially identical to those observed in other populations of the world. ALS accounted for $10 \%$ of the audlt death among Chamorros in the middle 1950 's. A form of parkinsonism associated with progressive dementia termed "parkinsonismdementia complex" is also endemic in this population. ${ }^{1}$

Neuropathologically PDC is characterized by severe neuronal loss affecting the cerebral cortex, especially the pyramidal neurons in the Ammon's horn and adjacent temporal cortex, and brain stem nuclei, especially the substantia nigra and locus ceruleus. ${ }^{2}$ Widespread Alzheimer's neurofibrillary changes are prominent features in the remaining neurons in the central nervous system. The topographic distribution of the tangles is remarkable in that it affects certain specific neurons, while sparing others. ${ }^{3}$ The most vulnerale neurons are the pyramidal cells in the Sommer's sector, glomeruli of parahippocampal gyrus (entorhinal cortex), amygdaloid nucleus, anterior olfactory nucleus, nucleus basalis of Meynert, various hypothalamic nuclei, substantia nigra, periaqueductal gray, dorsal raphe nucleus, locus ceruleus, and the reticular formation of the brain stem. Some neurons, such as the large anterior horn cells in the spinal cord, are only rarely affected and some other neurons such as Purkinje cells and primary sensory neurons are spared from the Alzheimer's neurofibrillary changes. It is noteworthy that unlike Alzheimer's disease the neurofibrillary tangles are not associated with senile plaque formation in PDC on Guam. Alzheimer's neurofibrillary changes are also observed in amyotrophic lateral sclerosis, although they are usually less extensive. ${ }^{3}$ The association of ALS and PDC is common and both disorders may occur in the same family. Although the etiology of ALS and PDC is still unknown, several hypothesis have been proposed.

During the past 20 years, the advent of immunohistochemical methods has made possible the evaluation of normal and abnormal structures at the molecular level and the identification of pathological changes permitted the use of specific markers. Our current immunohistochemical investigations were carried out on paraffin embedded clinical material from Guamanian patients 
that was collected over 25 years ago. In this communication, we will review our findings and compare them to observations made in various other neurodegenerative disorders.

\section{Ammon's Horn and Entorhinal Cortex}

\section{Synaptophysin}

Ammon's horn and entorhinal cortex are among the most severely affected areas of the brain in PDC and in Alzheimer's disease. The severe neuronal loss in Ammon's horn, especially Sommer's sector and subiculum is a characteristic feature of both diseases. A monoclonal antibody was used to visualize synaptophysin, an integral component of presynaptic vesicle membranes. ${ }^{4}$ We found a striking decrease of synaptophysin expression in the areas with neuronal loss in PDC and Alzheimer's disease. Synaptophysin reactivity was also diminished in the molecular layer of the dentate gyrus in both diseases, with the inner portion showing an almost normal, and outer portion a sharply reduced immunoreactivity. The input to the outer portion of the molecular layer of the dentate gyrus originates mainly from the entorhinal cortex and the septohippocampal projections are additional important extrinsic afferents to this molecular layer. The loss of the synaptophysin immunoreactivity seen in the outer molecular layer of the dentate gyrus in both diseases may be the result of the involvement of the entorhinal cortex and the septum in these conditions. ${ }^{4}$ The significant loss of afferents in certain hippocampal areas could be related to the memory impairment seen in these disorders. It is worthwhile to emphasize that in spite of the absence of senile plaque formation in PDC, there is a remarkable similarity to Alzheimer's disease with respect to synaptophysin expression and distribution.

\section{Neurofibrillary tangles}

Neurofibrillary tangles are most conspicuous microscopic features of the remaining neurons in PDC. With the use of conventional staining techniques and electron microscopy no apparent differences with regard to neurofibrillary tangles were observed in the Guam cases and Alzheimer's disease. ${ }^{5}$ We have now used immunohistochemical procedures to provide further information about the similarities in the two diseases. We investigated the expression of paired helical filaments, $\beta$ tau protein and ubiquitin by the tangles and our results were identical in both disorders. ${ }^{6}$ Studies regarding different morphological stages also revealed that the neurofibrillary tangles were similar in PDC and Alzheimer's disease. The same markers were also useful for detecting numerous neuropil threads (curly fibers or dystrophic axons) in the Sommer's sector and the subiculum in both diseases. In the region of the end plate of some PDC cases, abundant threads were observed. These novel observations provide additional evidence that support the view that the neurofibrillary tangles in PDC and Alzheimer's disease are indeed similar. Our findings indicate that despite the absence of senile plaques in PDC, this entity and Alzheimer's disease share to a considerable extent, common pathways that lead to cytoskeletal alterations. ${ }^{6}$

\section{$\beta$ amyloid protein-containing neurofibrillary tangles}

The lack of senile plaques was considered a remarkable histological feature on PDC on Guam. In our original investigations, carried out a quarter of a centruy ago, we used Bielschowsky silver impregnation and von Brownmühl stain on frozen section, Bodian stain on paraffin section, as well as Congo red stain for detecting Alzheimer's neurofibrillary tangles. With these classic conventional methods we clearly demonstrated the presence of abundant neuofibrillary tangles of various stages unassociated with senile plaque formation. Recently, however, the use of more sensitive silver stains, such as the modified Bielschowsky stains revealed in addition to the classic senile plaques, the presence of previously unrecognized amorphous argentophilic deposits which are referred to as diffuse plaques in patients with Alzheimer disease. Furthermore, immunohistochemical studies of $\beta$ protein (A4) confirmed that the diffuse plaques are amyloid deposit and probably represent an early phase in the formaion of senile plaques. ${ }^{7} \mathrm{By}$ applying the modified Bielschowsky stain to some recent cases of Guam PD and ALS, Dr Perl observed widespread, neocortical diffuse plaques in addition to the neurofibrillary tangles. ${ }^{7} \mathrm{He}$ suggested that the pathological spectrum of the lesion is moving toward that the incidence and pathological nature of the neurological disease on Guam. Indeed, a dramatic decline in the incidence of ALS on Guam has been reported. ${ }^{7}$ If this is true, it would be of extraordinary importance with regard to our ideas concerning the etiology of the disease. However, before we accept this conclusion, we have to consider a second possibility. Namely, that methods 25 years ago were inadequate to detect the changes discernible by modern techniques. Therefore, we reevaluated the material collected many years ago by using the modified Bielschowsky method and $\beta$-protein immunohistochemistry. This study revealed an absence of senile plaques in six out of the nine PDC cases analyzed. ${ }^{8} \mathrm{~A}$ few diffuse plaques were found scattered in the entorhinal and adjacent temporal cortex in the remaining two cases. In one case which had senile plaques, diffuse plaques were also detected in molecular layer of the dentate gyrus, the Sommer's sector of the hippocampus and the subiculum. No amyloid deposit was 
seen in the hippocampus of the other eight PDC cases. The three cases in which amyloid deposits were found corresponded to the oldest individuals in the series (over age 61). It should be mentioned here that patients with bona fide Alzheimer's disease have been identified among Chamorros in Guam. ${ }^{7}$ These rare cases have the typical histological features of Alzheimer's disease without neuronal loss in the substantia nigra. Abundant senile plaques are not only present in their cerebral cortex, without neuronal loss in the substantia nigra. Abundant senile plaques are not only present in their cerebral cortex, but also in the basal ganglia and cerebellum. As in Alzheimer's disease, elsewhere, the plaques are $\beta$ protein positive and prion negative. ${ }^{9}$ During the immunohistochemical survey of $\beta$ protein in PDC on Guam, amyloid protein-containing neruofibrillary tangles ( $\beta$-NFTs) were found in the temporal lobes of the three oldest individuals. ${ }^{8}$ The $\beta$-NFTs were most frequent in the entorhinal cortex and senile plaques were invariably observed in their vicinity (Fig 1 ). On the other hand, in the regions without senile plaques, even though abundant neurofibrillary tangles were recognized with the modified Bielschowsky method, no $\beta$-NFTs were found. The density of $\beta$-NFTs seemed to be proportional to that of senile plaques and none were observed in cases lacking senile plaques. These data suggest that $\beta$-NFTs result from a secondary amyloid deposition on certain neurofibrillary tangles.

\section{Basal Ganglia and Substantia Nigra}

The basal ganglia are well known regions involved in

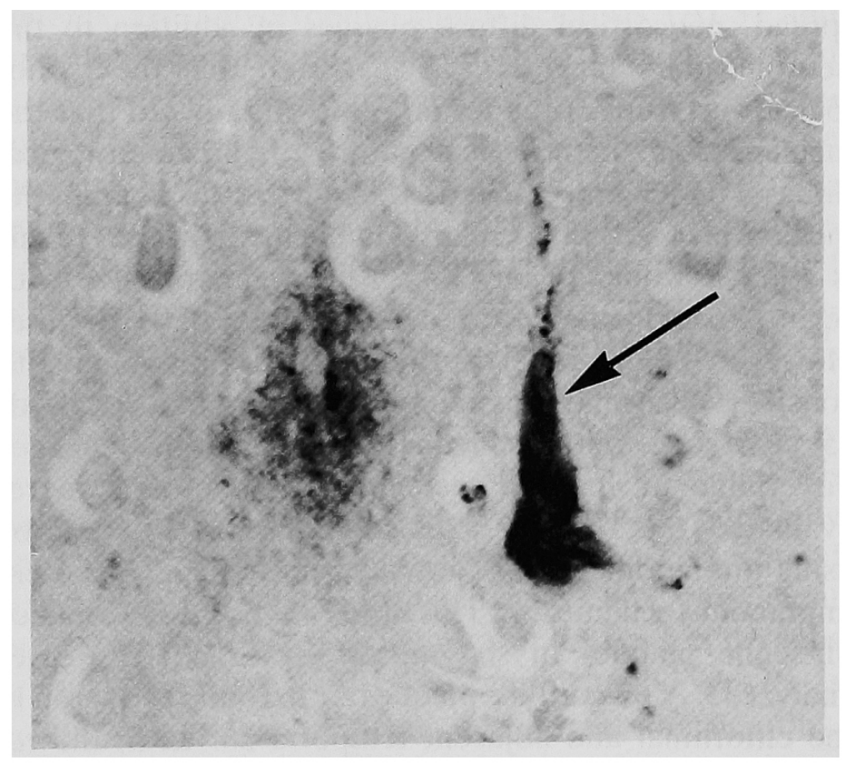

Fig 1 A pyramid shaped neurofibrillary tangle stained with the antibody to $\beta$-protein (arrow) and a diffuse senile plaque in the entorhinal cortex of a patient with parkinsonism-dementia complex on Guam. $\times 500$.
Parkinson's disease, Huntington's disease, progressive supranuclear palsy, striatonigral degeneration and other extrapyramidal diseases. In contrast to certain entities, such as Huntington's disease and striatonigral degeneration which exhibit a distinct gross pathology of the basal ganglia, by using routine neuropathological staining methods no such striking basal ganglia alterations have been described in Parkinson's disease. However, the application of immunohistochemical procedures to the study of the basal ganglia and the substantia nigra provided importnat new infomation. Thus, the striatal projection neurons and their efferent fibers were analyzed with antibodies to calcineurin, Met-enkephalin and substance $\mathrm{P}^{10}{ }^{10}$ Tyrosine hydroxylase served as a marker for nigrostriatal dopaminergic neurons. ${ }^{10}$ The basal ganglia of patients with PDC reacted strongly with each antibody and in all instances a normal distribution pattern was seen. ${ }^{10}$ Furthermore, the striosomal organization was identified in Parkinson's disease ${ }^{11}$ and in PDC $^{12}$ (Fig 2). These findings indicate that the striatal output system is well preserved in PDC patients. Similar findings were obtained in patients with Alzheimer's disease or Parkinson's disease. However, as compared to the patients with Alzheimer's or Parkinson's disease, the patients with PDC revealed a severe loss in the number of dopaminergic neurons in both, the medial and the lateral portions of the substantia nigra. ${ }^{10}$ In view of the functional corticosubcortical loops, these findings could explain, in part, the cognitive impairment and the Parkinsonian features that occur in PDC on Guam.

In patients with progressive supranuclear palsy, a severe depletion of substance $P$ and Met-enkephalin-like immuno-reactivities was observed in the internal and



Fig 2 Met-cnkephalin positive striosomes in the striatum in a patient with parkinsonism-dementia complex on Guam. $\times 350$. 


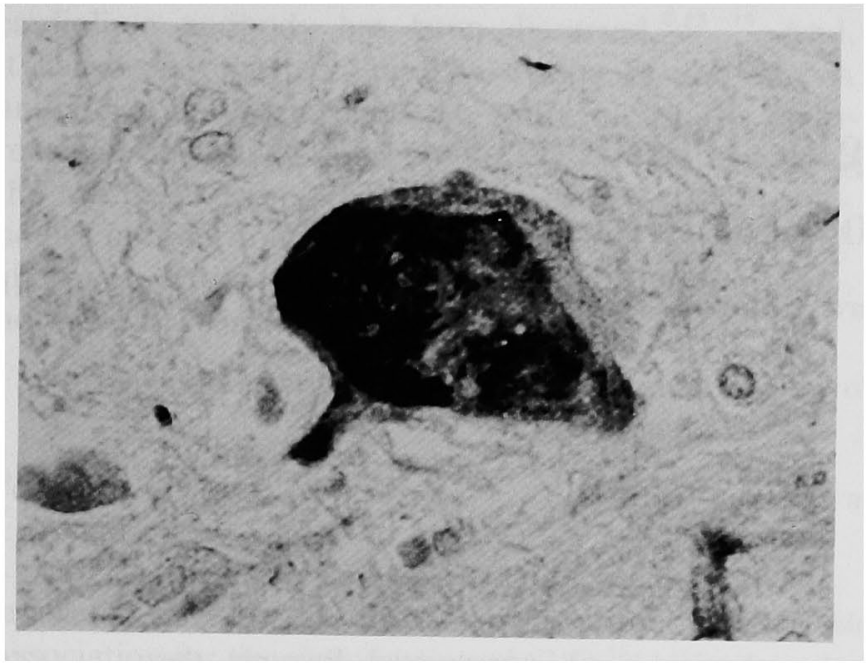

iig 3 Neurofibrillary tangle detected with the anti-tau antibody in the pinal cord of a patient with parkinsonism-dementia complex on Guam. $<800$.

xternal segments of the globus pallidus, respectively. ${ }^{13,14}$ These findings corroborate the pronounced degenerttion of pallidal neurons demonstrable by conventional listological staining.

\section{Spinal Cord}

One of our recent noteworthy findings pertains to cal deposits of ubiquitin in the anterior horn cells $f$ the spinal cord of patients with ALS, ${ }^{15}$ both of the poradic and familial types and its absence in control 1dividuals. Ubiquitin deposits are also observed in juam ALS. ${ }^{16}$

Since Alzheimers neurofibrillary tangles are seen in he spinal cord of Guam ALS and PDC, we investigated au protein expression in the spinal cord in these patients. $V i t h$ the immunohistochemical technique employed we iere able to localize the neurofibrillary tangles (Fig 3) 1ore readily than by using conventional methods. ${ }^{17}$ In ontrast to neurons in the lateral marginal areas of the osterior horn, only occasional Alzheimers neurofibrilrry tangles were seen in large anterior horn cells. The eurofibrillary tangles are intracellular and are not nly confined to soma, but are also found in neuronal rocesses similar to those in the cerebral cortex. The eurofibrillary tangles in the spinal cord were filaments omposed of around 15-nm-wide straight fibrils and few paired helical filaments with a periodicity of aproximately $80 \mathrm{~nm} .{ }^{18}$ Alzheimers neurofibrillary tangles rere observed in all Guamanian ALS and PDC cases xamined, but none was seen in non Guamanian ALS.

cknowledgments: The author expresses his sincere appreciation to ir F Herz for reviewing the manuscript. This work was supported in art by the Amyotrophic Lateral Sclerosis Association.

\section{References}

1. Hirano A. Kurland LT, Krooth RS, Lessell S: Parkinsonismdementia complex: an endemic disease on the island of Guam. I. Clinical features. Brain 1961, 84: 642-661

2. Hirano A, Malamud N, Kurland LT: Parkinsonism-dementia complex, an endemic disease on the island of Guam. II. Pathological features. Brain 1961, 84: 662-679

3. Hirano A, Zimmerman HM: Alzheimers neurofibrillary changes: a topographic study. Arch Ncurol 1962, 7: 227-242

4. Goto S, Hirano A: Neuronal inputs to hippocampal formation in Alzheimer's disease and in parkinsonism-dementia complex on Guam. Acta Neuropathol 1990, 79: 545-550

5. Hirano A, Dembitzer HM, Kurland LT, Zimmerman HM: The fine structure of some intraganglionic alterations: neurofibrillary tangles, granulovacuolar bodies and "rod-like" structures as seen in Guam amyotrophic lateral sclerosis and parkinsonism-dementia complex. J Neuropathol Exp Neurol 1968, 27: 167-182

6. Ito H, Goto S, Hirano A, Yen S-H: Immunohistochemical study of the hippocampus in parkinsonism-dementia complex on Guam. J Geriat Psych Neurol (in press)

7. Hirano A: ALS on Guam. In pursuit of pathology over 30 years. In: Rose FC, Norris F. cds, Amyotrophic Lateral Sclerosis. New Advances in Toxicology and Epidemiology, London, SmithGordon, 1990, 100-111

8. Ito $\mathrm{H}$, Hirano $\mathrm{A}$, Yen S-H, Kato $\mathrm{S}$ : Demonstration of $\beta$ amyloid protein-containing neurofibrillary tangles in parkinsonismdementia complex on Guam. Neuropathol Appl Neurobiol 1991, 17: $365-373$

9. Suenaga T, Hirano A, Llena JF: Cerebellar senile plaques in a Chamorro patient on Guam. Shinkei Naika (Neurol Med) 1990 , 33: $523-524$ (in Japanese)

10. Goto S, Hirano A, Matsumoto S: Immunohistochemical study of the striatal efferents and nigral dopamingeric neurons in parkinsonism-dementia complex on Guam in comparison with those in Parkinsons and Alzheimers diseases. Ann Neurol 1990. 27: $520-527$

11. Sakamoto S, Goto S, Ito H, Hirano A: Striosomal organization of substance P-like immunoreactivity in Parkinsonian patients. Neurology (in press)

12. Ito $\mathbf{H}$, Goto $\mathrm{S}$, Sakamoto $\mathrm{S}$. Hirano A: Striosomal organization in parkinsonism-dementia complex on Guam. An immunohistochemical study (submitted for publication)

13. Matsumoto S, Goto S. Mizusawa H, Hirano A: Immunohistochemical studies of substance $P$ and Met-enkephalin in the globus pallidus of progressive supranuclear palsy. In: Yoshida M. ed, Alzheimers and Parkinsons Discases, New York, Plenum Press. (in press)

14. Matsumoto S, Goto S, Hirano A: A comparative immunohistochemical study on striatal Met-cnkephalin expression in Alzheimcrs disease and in progressive supranuclear palsy. Acta Neuropathol 1990, 81: 74-77

15. Hirano A: Cytopathology of amyotrophic lateral sclcrosis. Adv Neurol 1991, 56: 91-101

16. Matsumoto $S$, Hirano $A$, Goto $S$ : Ubiquitin-immunoreactive filamentous inclusions in anterior horn cells of Guamanian and non-Guamanian amyotrophic lateral sclerosis. Acta Neuropathol 1990, 80: $233-238$

17. Matsumoto S, Hirano A, Goto S: Spinal cord neurofibrillary tangles of Guamanian amyotrophic lateral sclerosis and Parkinsonismdementia complcx: an immunohistochemical study. Neurology 1990, 40: 975-977

18. Kato S, Hirano A: Ultrastructural identification of Alzhcimers ne urofibrillary tangles (NFT) in spinal cord in Guamanian patients with amyotrophic lateral sclerosis (ALS) and parkinsonismdementia complex (PDC). J Neuropathol Exp Neurol 1991. 50: 306 